\title{
Magnetic resonance imaging in inflammatory rheumatoid diseases
}

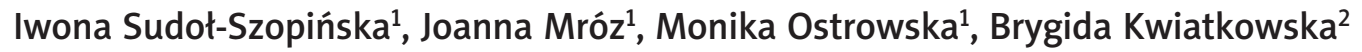 \\ ${ }^{1}$ Department of Radiology, National Institute of Geriatrics, Rheumatology and Rehabilitation, Warsaw, Poland \\ ${ }^{2}$ Early Arthritis Clinic, National Institute of Geriatrics, Rheumatology and Rehabilitation, Warsaw, Poland
}

\begin{abstract}
Magnetic resonance (MR) is used more and more frequently to diagnose changes in the musculoskeletal system in the course of rheumatic diseases, at their initial assessment, for treatment monitoring and for identification of complications. The article presents the history of magnetic resonance imaging, the basic principles underlying its operation as well as types of magnets, coils and MRI protocols used in the diagnostic process of rheumatic diseases. It enumerates advantages and disadvantages of individual MRI scanners. The principles of MRI coil operation are explained, and the sequences used for MR image analysis are described, particularly in terms of their application in rheumatology, including T1-, T2-, PD-weighted, STIR/TIRM and contrast-enhanced T1-weighted images. Furthermore, views on the need to use contrast agents to optimise diagnosis, particularly in synovitis-like changes, are presented. Finally, methods for the assessment of MR images are listed, including the semi-quantitative method by RAMRIS and quantitative dynamic examination.
\end{abstract}

Key words: rheumatoid arthritis, magnetic resonance imaging, rheumatic inflammatory diseases, MRI protocol.

\section{Introduction}

Magnetic resonance imaging (MRI) was introduced to the classification criteria of axial spondyloarthropathies in 2009 [1]. As of today, it is the only group of rheumatic diseases with MRI findings included in its criteria. As for other disease entities (mainly systemic connective tissue diseases), the diagnosis, apart from clinical and laboratory data, is based on radiographs, which, as is widely known, specifically present only late pathological changes.

Despite this, MRI is more and more common in rheumatic patients at early stages of the diagnosis, for treatment monitoring, early detection of complications as well as in confirming remission [2]. In spondyloarthritides, MRI findings can be the basis for implementing biological treatment.

The article presents the basic principles underlying MRI operation as well as types of magnets, coils and MRI protocols used in the diagnostic process of rheumatic diseases, particularly rheumatoid arthritis.

\section{Magnetic resonance imaging hardware and magnets}

Magnetic resonance imaging is based on the wellknown physical phenomenon of resonance, in this case magnetic resonance, i.e. stimulation, by radio waves (RF), of protons (hydrogen atoms) placed in an adequately spatially shaped magnetic field of a stable Bo field value. The frequency of these waves is consistent with the resonance frequency of the scanned area and shows linear dependence on the strength of the Bo magnetic field. $A$ radiofrequency coil (RF coil) which generates an additional magnetic field (B1) perpendicular to Bo is both the receiver and transmitter of an MRI scanner. Frequently, it is also the "parent coil." Depending on the part of the body examined, coils used in MR imaging differ in their size, shape, basic construction, i.e. number of channels, manner of their connection and, most importantly, frequency, i.e. RF signal emission.

\footnotetext{
Address for correspondence:

Iwona Sudoł-Szopińska, Department of Radiology, National Institute of Geriatrics, Rheumatology and Rehabilitation, Spartanska 1, 02-637 Warsaw, Poland, e-mail: sudolszopinska@gmail.com

Submitted: 27.06.2016; Accepted: 30.08.2016
} 
Magnetic resonance imaging scanners are broadly classified into high-field whole-body MRI units (WB-MRI) or dedicated extremity units (E-MRI) of low-to-mid field strength. The WB-MRI units of the high field of 1.5 up to $3 \mathrm{~T}$ are of a closed bore cylindrical design with superconductive magnets, kept at superconducting temperatures. They create a stable homogeneous magnetic field, stabilised by solenoid coils bathed in liquid helium.

Area-dedicated E-MRI units contain an air gap between two magnetic poles. They may utilise permanent magnets or electromagnets with a permanent C-shaped magnet that operates at low-to-mid field strength of 0.2 to $1 \mathrm{~T}$, or dipolar electromagnets with coils on either side of the patient. These coils can be superconductive or resistive and range from $0.2 \mathrm{~T}$ to $1.2 \mathrm{~T}$. In addition, preclinical research and mainly animal studies utilise units of high fields of 4.7 T, 7.0 T, 9.4 T, 11.7 T and even very high, ultra-high fields of $15.2 \mathrm{~T}$, which are not yet approved by the FDA [3].

The advantages of WB-MRI units [4] indicate their 90\% superiority over E-MRI units.

Disadvantages of low-field scanners, which result in their rare usage, include [5]:

- a small field of view (FOV), limited to approximately $12 \mathrm{~cm}$ (e.g. only the wrist and metacarpophalangeal (MCP) joints can be examined at one setting),

- low signal-to-noise ratio (SNR), as it is approximately proportional to the field strength,

- longer examination to maintain equivalent signal-tonoise ratio,

- significantly less homogeneous main magnetic field, resulting in poor image quality,

- reduced detection of gadolinium enhancement (gadolinium enhancement is less apparent at lower fields, which means that relatively higher doses of gadolinium must be administered for the same relative enhancement),

- impaired detection of calcifications, iron accumulation and haemorrhage,

- reduced number of possible imaging techniques compared to whole-body units, especially the lack of selective fat saturation sequences (FS/SPAIR) $[6,7]$.

Fat suppression (FS) requires a homogeneous field and high magnetic field strengths, which are not available in low-field units [6]. The only FS technique possible in E-MRI units is short T1 inversion recovery (STIR), based on relaxation time differences, which can provide comparable information on bone marrow oedema (BME), although with fewer details [6]. However, new E-MRI units are emerging, operating at 1-1.2 T field strength.

Advantages of low-field scanners include:

- open design that maximises patient comfort and minimises claustrophobia,
- access to the patient for interventional MRI procedures and anaesthesia, where monitoring equipment can be brought closer to the low-field scanner and to the patient,

- less expensive system and operation (lower-field permanent scanners do not require liquid helium or special maintenance),

- reduced projectile risk to patients and personnel,

- reduced susceptibility-induced spatial distortions and signal loss when scanning patients with metal hardware, compared to those occurring at high fields,

- the amount of energy deposited in tissues by radio frequency pulses (the specific absorption rate, SAR) is low (as it is proportional to the square of magnetic field strength).

\section{Coils}

Magnetic resonance imaging of specific anatomical regions, including the spine, wrist, hand, knee, ankle, foot, finger as well as head, breast etc., is performed using dedicated RF coils of different designs. The hand and wrist, followed by the foot and ankle and cervical spine, are the most frequent locations of rheumatoid arthritis (RA), and, at the same time, these regions are the most frequently scanned by MRI in RA patients. That is why the most frequent coils used in rheumatic patients are: a wrist coil, foot and ankle coil and coils for spine imaging (e.g. spine coil and head and neck coil). Other coils used are: knee, shoulder, elbow, XL torso and cardiac coils (for hip, SIJ imaging), as well as Q-body coils for whole body imaging (Fig. 1).

Various strategies may be employed for MR imaging of the hand and wrist in patients with early arthritis [8]. As for coil selection, dedicated wrist coils, flexible coils, knee coils, hand and neck coils and surface coils (for finger joints) are in use. Dedicated wrist coils provide high quality images and high comfort of examination; the examined hand is positioned along the body (although in obese patients the superman position is required). In addition, using dedicated wrist coils, the distal radius and ulna, the carpal bones and the MCP joints can be visualised together within an FOV of 120-160 mm. In order to assess proximal and distal interphalangeal joints, and sometimes even MCP joints (in patients with large hands MCP joints can be excluded because of a small FOV), another MRI examination is needed.

In early RA, hand and wrist involvement is usually bilateral. Therefore, some centres perform bilateral MR imaging of the hands and wrists, whilst others prefer to study only the dominant or more painful hand and wrist, assuming that joint involvement will be lower on the contralateral side [8]. Scanning of both hands would be optimal in RA, but is often not feasible with adequate 

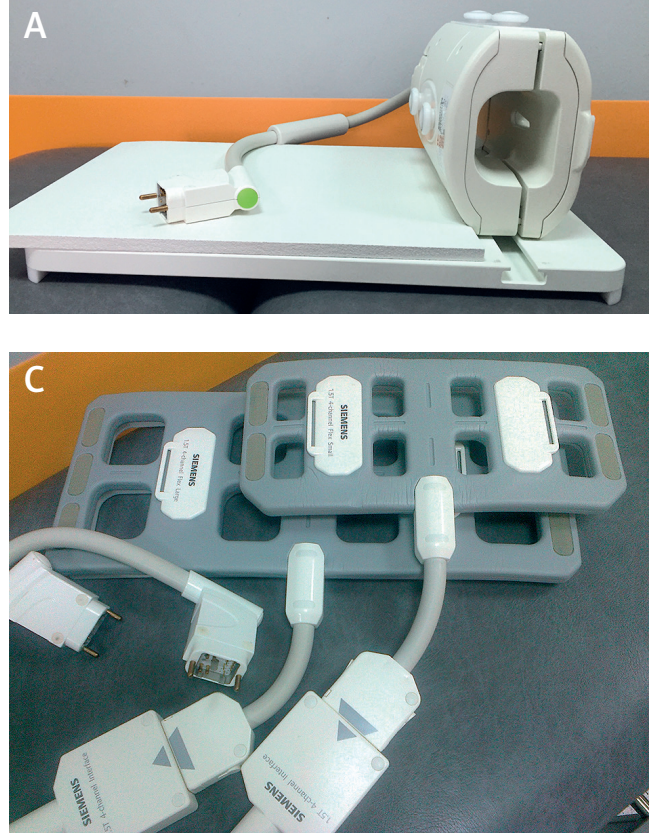

Fig. 1. Examples of coils used in MRI in patients with RA: A) wrist coil; B) foot and ankle coil; C) flex coils; D) knee coil.

image quality (hands above the head folded in prayer in a knee or surface flex coil, or hands side by side in a knee, head and neck coil or flex coil in an uncomfortable position on the abdomen). If indicated, WB-MRI scans may also be acquired when looking for active inflammation. However, the readability of WB-MRI is low for the elbows and interphalangeal joints. Therefore, it is a suboptimal approach but quick and useful for general consideration of implementing treatment [9] (Fig. 2).

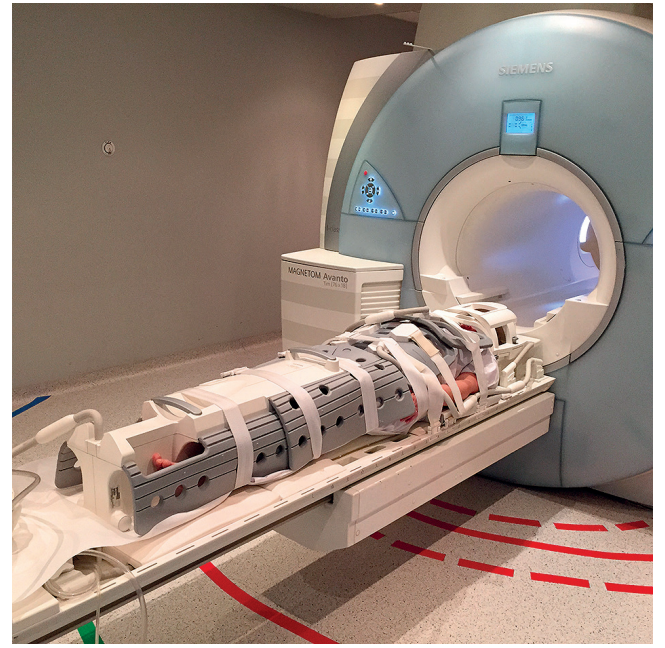

Fig. 2. WB-MRI
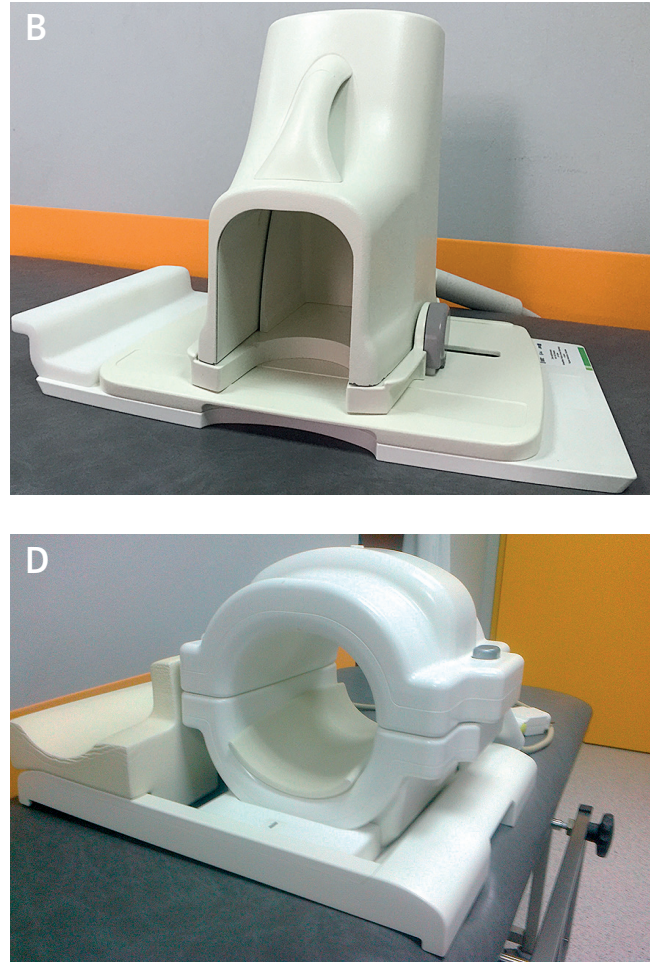

Foot and ankle coils are excellent. However, these structures are well seen in a knee coil as well. MRI of the cervical spine is done in a spine coil or head and neck coil. The figures below (Fig. 3) show the various methods of application of the coils used in hand and wrist MRI imaging and corresponding MRI scans (Fig. 4).

\section{Sequences and protocols}

Magnetic resonance imaging protocols should be adjusted taking into consideration scanner specifications, available coils and image quality in particular sequences [2].

The recommended maximal slice thickness is $3 \mathrm{~mm}$ with an interslice gap of $0.3 \mathrm{~mm}$, depending on the anatomical structure assessed. Thinner sections should ideally be performed for small joints [2], especially of the hands. For large joints (knee, shoulder, hip, ankle), the $3 \mathrm{~mm}$ thickness should be used.

The following sequences are subject to assessment: T1-, T2- and PD-weighted with or without FS or STIR/TIRM sequences as well as available post-contrast ones. They enable the diagnosis of pathological changes within the synovium, subchondral bone marrow and adipose tissue [2].

T1-weighted images are favoured due to their relatively short imaging duration, good presentation of anatomical details and their ability to visualise the inflamed synovium after i.v. contrast injection (gadolinium, Gd, 
Fig. 3. Bilateral hand and wrist MRI examination: A) hands folded in prayer position in a head and neck coil; B) hands placed side by side in a body matrix coil.
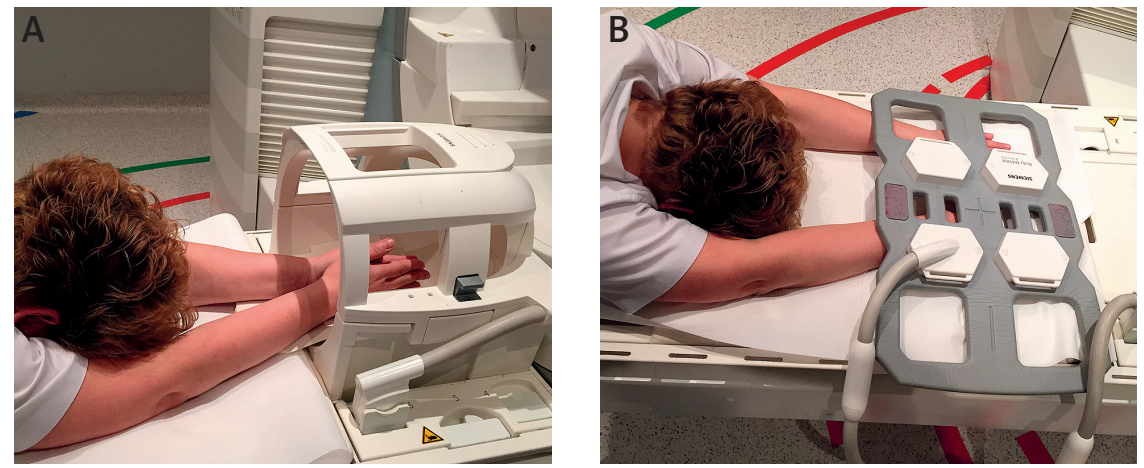

post-Gd images). Fat and Gd-enhanced tissue has a high signal intensity on T1-weighted images and, since Gd uptake depends on tissue vascularity and perfusion, the highly vascularised and perfused inflamed synovium is easily recognisable [6]. In T1FS post-Gd images, FS increases the contrast between the inflamed synovium and adjacent structures [6]. This make inflammatory changes more easily recognisable.

T2-weighted images depict both fat and fluid/oedematous tissues with a high signal intensity. These are particularly useful when fat saturation techniques, in which the signal from fat is suppressed, are applied [8]. T2-weighted FS increases the detection of oedema/fluid located in areas with fatty tissues, e.g. bone marrow oedema [6].

$\mathrm{STIR}$ is a fat suppression technique. Inversion-recovery imaging allows homogeneous and global fat suppression. However, it is not specific for fat, and the signal intensity of tissues with long $\mathrm{T} 1$ and tissues with short T1 may be similar.

PD FS, like T2 FS (the saturation technique is the same in both sequences) and IR sequences (STIR/TIRM a different way of fat saturation), are all sensitive to the detection of fluid, although these results are obtained by different mechanisms. PD gives a higher signal-to-noise ratio (SNR), while T2 and IR are more sensitive to fluid.

T1 FS post-contrast images are used to increase the specificity of MRI diagnosis, mainly to discriminate between exudate and synovitis or tenosynovitis. They are not really necessary for assessment of erosions and bone marrow oedema (BME) [10]. Also, there have been studies evaluating the real need to perform contrast-enhanced MRI for peripheral joint evaluation, bearing in mind that the use of intravenous Gd is an invasive procedure, adds to the overall examination time and cost and may, although rarely, induce side effects (it carries a small risk of nephrogenic systemic fibrosis, exclusively in patients with impaired renal function, i.e. GFR $<30 \mathrm{ml}$ $\left.\min / 1.73 \mathrm{~m}^{2}\right)[5,10]$. Also, some MRI sequences, such as T2-weighted FS and STIR, display areas with high water content as bright areas. Thus, oedematous areas within
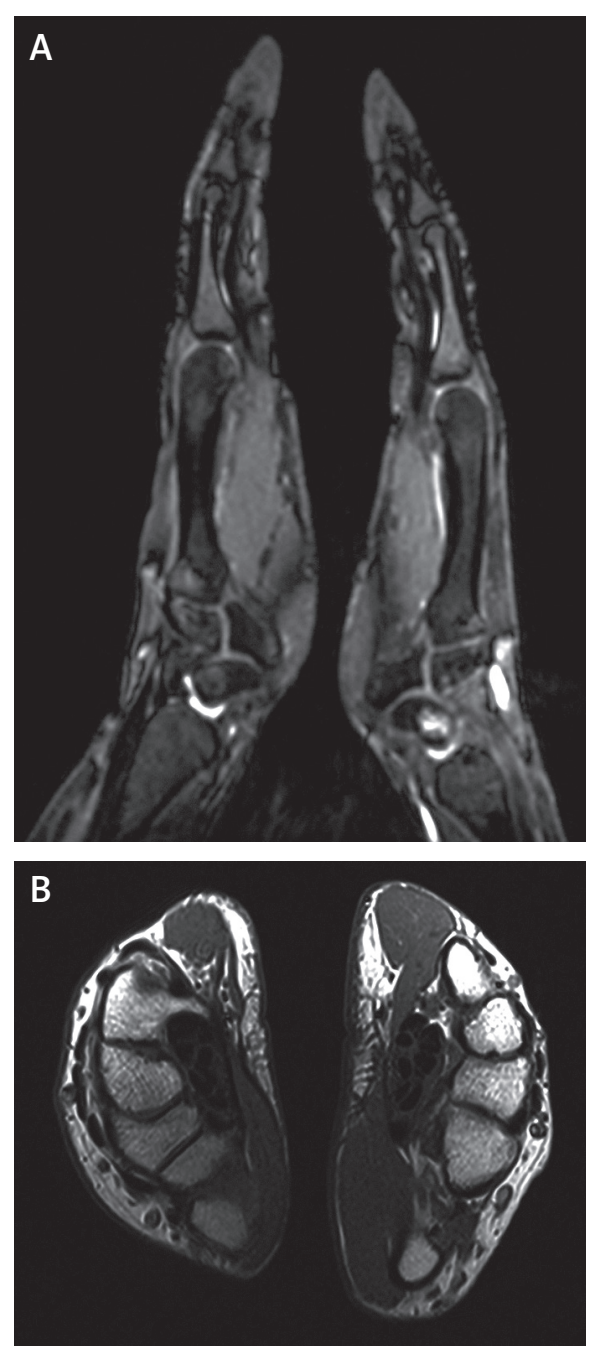

Fig. 4. Corresponding MR image of both hands (A, B).

the inflamed synovium show increased signal intensity in such fluid-sensitive sequences. Moreover, the evaluation of synovitis of the wrist and joints in MRI with no contrast injection has been reported [5]. Nevertheless, to evaluate synovitis and tenosynovitis in RA, intravenous 
administration of paramagnetic gadolinium-containing contrast agent is generally recommended to make the inflamed synovium easy to recognise and evaluate [5].

Stomp et al. [10] have proven that omitting Gd-based contrast agent administration results in low specificity for synovitis and low sensitivity for tenosynovitis, which indicates that Gd contrast administration remains essential for an optimal diagnosis. Another important advantage of using Gd contrast is the ability to differentiate between effusion and inflammation. Bearing in mind that even a small physiological amount of fluid may give a false appearance of synovitis on T2 and STIR images, which will negatively affect specificity, post-contrast MR images are needed [10].

As for BME, Stomp et al. [10] reached an excellent agreement between T2 images and T1Gd sequences: the sensitivity and specificity of both sequences for the detection of BME were equally high. Because contrast is usually administered to assess (teno)synovitis, a short protocol of T1 and T1Gd has been proposed by the authors as sufficient in RA (T2 sequences take approximately 4 min - $20 \%$ of the duration).

The RA MRI Scoring (RAMRIS) system, a standardized semi-quantitative assessment of inflammatory soft tissue and destructive bone alteration, recommends that imaging should include T1-weighted sequences primarily to assess erosions, T2-weighted FS or STIR to evaluate BME and post-gadolinium T1Gd in combination with non-contrast T1-weighted images to evaluate synovitis and tenosynovitis [10,11]. The RAMRIS does not describe the use of FS for post-contrast T1. In practice, however, it is commonly used to increase the visibility of enhancement and to differentiate enhancement from fatty tissue on fast spin echo sequences which exhibit a high signal of fat [11]. Thus, the standardised use of fat suppression in T1Gd sequence aids in identifying enhancing BME in the fatty bone marrow. In addition, better image quality favours T1Gd sequences [12]. One limitation of using only T1Gd sequences is that small effusions, bright on $\mathrm{T} 2$ but not enhancing, may be harder to detect [11].

Other highlights in rheumatology are very thin, $1.5 \mathrm{~mm}$, isotropic (the same voxel volume in every dimension), 3D, fluid-sensitive, T2-weighted images and $1.0 \mathrm{~mm}$ post-contrast and subtraction 3D images. They can be useful for both initial diagnosis and follow-up imaging, allowing multiplanar reconstructions (MPR) to ensure the achievement of comparable planes, even if patient positioning has not been exactly reproduced. MPR of 3D images allows images to be created from the original axial plane in the coronal, sagittal or oblique plane. Examples of using dynamic contrast are shown below in Figure 5.

\section{Assessments of magnetic resonance images}

The assessment of MR images is usually qualitative. There are several time-consuming semi-quantitative methods for RA and psoriatic arthritis which, however, are mostly used in research studies [2]. The only validated method is the RAMRIS devised by the OMERACT group for the wrist and MCP joints. However, the results for inter-reader correlation show substantial variation between studies. Moreover, this system is moderately reliable and sensitive, has poor sensitivity to synovial changes and is more sensitive to early erosions than radiographs, but not to long-term progression [13]. In 2007, it was supplemented with semiqualitative assessment of tenosynovitis at the wrist in 10 anatomical areas.

With quantitative methods, inflammatory activity can be estimated by quantifying an increase in early synovial signal intensity in dynamic CE MR imaging (DCE-MRI) [8].

\section{Discussion}

Magnetic resonance imaging has become an important diagnostic tool in the evaluation of rheumatic diseases $[12,14,15]$. It is currently considered the best non-invasive, observer-independent imaging modality to evaluate inflammation of joints, tendons, entheses and bone marrow in adults, children and adolescents with rheumatic conditions [2]. According to the Recommendation of the Arthritis Subcommittee of the European Society in Musculoskeletal Radiology [2], MRI allows the:

- assessment of peripheral joints for active inflammation in the form of effusion, synovitis, bone marrow oedema, as well as the subsequent structural lesions, such as articular surface damage and cortical bone erosions,

- assessment of active inflammatory lesions and structural changes in the sacroiliac joints,

- assessment of inflammatory and post-inflammatory lesions of the vertebral joints, i.e. assessment of the inflammatory activity, aseptic spondylodiscitis, atlanto-axial / atlanto-occipital structural lesions,

- assessment of tenosynovitis and enthesopathic lesions,

- confirmation of clinical diagnosis based on imaging characteristics and/or location of lesions,

- qualitative, semi-quantitative and quantitative measurements of active inflammation and chronic joint damage.

As far as rheumatic diseases are concerned, MRI surpasses plain radiography and ultrasonography with its ability to visualise the bone marrow involvement. Also, early inflammatory features within soft tissues (joints, tendons sheaths and bursae, muscles) that are not seen on plain radiography in detail and are inaccessible to ultrasound (e.g. of the spine, sacroiliac joints) or their 

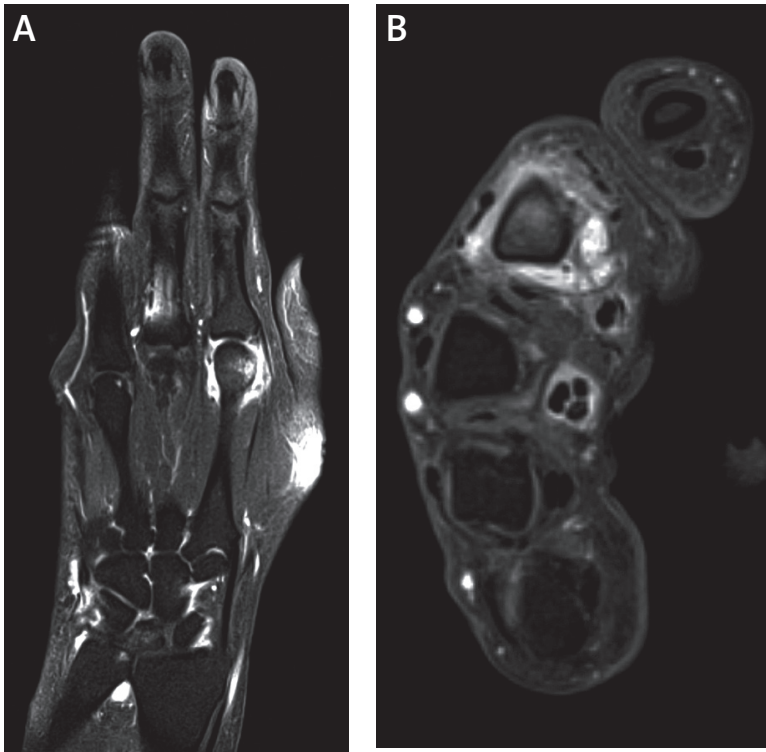

Fig. 5. Dynamic contrast-enhanced $M R I$ of the hand: T1FSCM in coronal (A), axial (B) planes and dynamic contrast-enhanced sequences (C, D).

assessment in ultrasound is limited (e.g. hip and gleno-humeral joints) are well seen in MRI.

The limitations of MRI include [16]:

- long examination time,

- high cost,

- necessity to inject contrast in order to increase specificity,

- metallic implants, which might be moved or whose function might be affected by a strong magnetic field,

- inability to assess osteoporosis.

With regard to osteoporosis, which is frequently seen in rheumatic disease, dual x-ray absorptiometry remains the state-of-the-art technique to measure bone mineral density. MR application, to date, is a subject of promising research, including ultrashort-echo-time (UTE) imaging techniques for quantification of water content of the cortical bone to assess bone quality, proton MR spectroscopy for quantifying marrow adiposity, MR spectroscopy providing quantitative assessment of water and fat content in bone marrow and information on different compartments of lipids in marrow, and finally dynamic contrast-enhanced MR imaging to be used to measure perfusion indexes [17].

In addition, MR images are in many cases non-specific and require differentiation from other pathologies manifested by the same spectrum of changes. Apart from
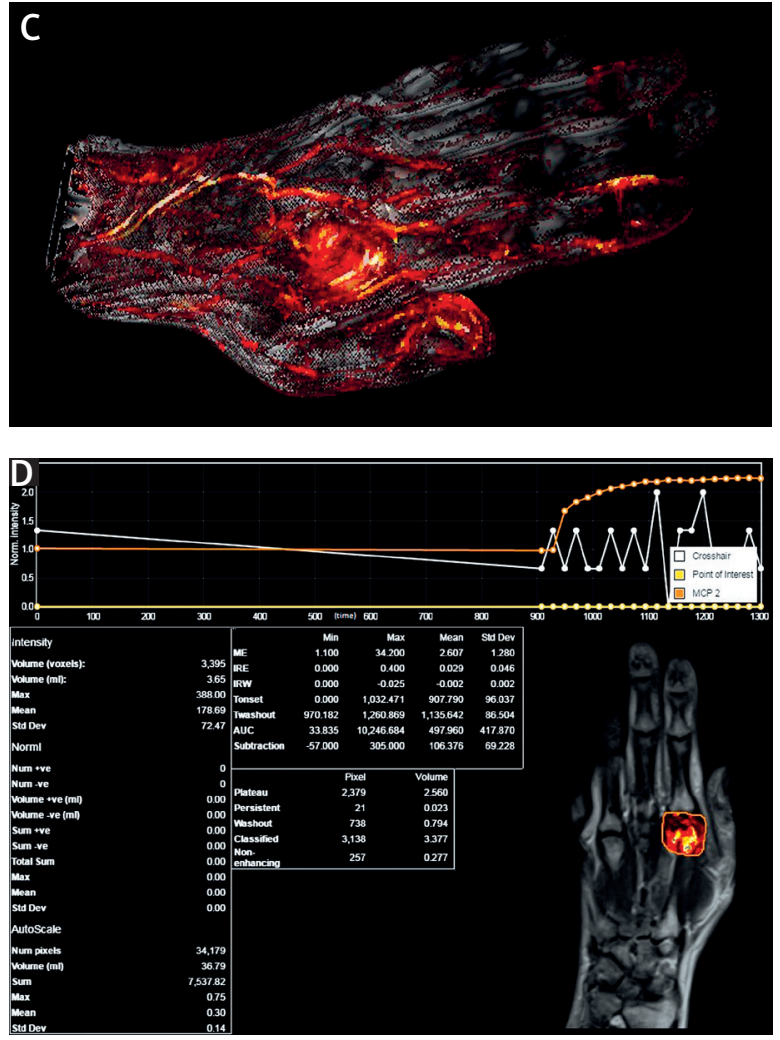

other inflammatory entities, which are frequently differentiated by clinical and laboratory findings, BME may be encountered in a number of other pathologies, i.e. [18]:

- overuse lesions,

- neoplasms,

- post-traumatic lesions,

- bone necrosis,

- polyneuropathy,

- leukaemia and other myeloproliferative lesions,

- bone marrow reconversion following chemotherapy. In order to establish the final diagnosis, it is necessary to obtain complete and comprehensive information from the referral concerning the underlying condition, its treatment, comorbidities as well as the history of injuries and surgical procedures, particularly in the region to be examined. Such referral will ensure proper interpretation of MRI findings and can help to minimize false positive diagnoses.

Nevertheless, MRI findings alone in many cases are specific and enable a proper differential diagnosis. These include:

- location of BME (such as subchondral in spondyloarthropathy),

- involvement of bone and soft tissues, with abscess formation (in infections),

- injuries, especially overuse, 
- soft tissue traumatic lesions,

- neoplasms, with precise delineation of the extent of any lesion and perfusion by MRI imaging,

- fatty involution (atrophy) of muscles.

\section{Summary}

The Arthritis Subcommittee of the ESSR recommends MRI as an accurate and objective modality for the diagnosis and follow-up of rheumatic conditions. The main indications for MRI in patients with musculoskeletal rheumatic diseases include [2]:

- early diagnosis of inflammation in both soft tissues and bone marrow, before destructive lesions develop (cysts, erosions, cartilage damage),

- confirmation of the presence of clinically active changes and post-inflammatory structural lesions,

- disease follow-up, including monitoring of therapy response,

- identification of disease complications.

In particular [2]:

- For rheumatoid arthritis and juvenile idiopathic arthritis MRI is the best method to assess cervical spine involvement and early inflammatory lesions in other tissues/joints that are not detected on clinical examination (e.g. temporomandibular joints and sacroiliac joints in juvenile idiopathic arthritis). Contrary to radiographs and ultrasound, MRI may visualize BME, which is considered a pre-erosive stage and could be the only location of the disease, as well as subtle/initial stages of synovitis, e.g. in the above-mentioned joints.

- In axial spondyloarthritis MRI plays an important role in detecting sacroiliitis (ASAS imaging arm) more sensitively and also with better reproducibility compared to conventional radiography, and it is recommended particularly in young patients or patients with a short history of disease, or where clinical findings and conventional radiography fail to definitively confirm the disease.

- In peripheral spondyloarthritis and other entities with peripheral joint involvement, MRI may visualize BME and soft tissue inflammation and is indicated when conventional radiographs and/or ultrasound findings are inconclusive or normal.

The authors declare no conflict of interest.

\section{References}

1. Sieper J, Rudwaleit M, Baraliakos X, et al. The assessment of SpondyloArtritis internationalSociety (ASAS) handbook: a guide to assess spondyloarthritis. Ann Rheum Dis 2009; 68; 1-44.

2. Sudoł-Szopińska I, Jurik AG, Eshed I, et al. Recommendations of the ESSR Arthritis Subcommittee for the use of magnetic resonance Imaging in musculoskeletal rheumatic diseases. Semin Musculoskelet Radiol 2015; 19: 396-411.

3. https://www.bruker.com/fileadmin/user_upload/8-PDF-Docs/ MagneticResonance/MRI/brochures/152-11_mouse_T138908.pdf

4. Cosmos TC, Parizh M. Advances in whole-body MRI magnets. IEEE Trans Appllied Superconductivity 2011: 21; 2014-2019.

5. Eshed I, Krabbe S, Østergaard M, et al. Influence of field strength, coil type and image resolution on assessment of synovitis by unenhanced MRI-a comparison with contrast-enhanced MRI. Eur Radiol 2015; 25: 1059-1067.

6. Østergaard M, Duer A, Moller U, Ejbjerg B. Magnetic resonance imaging of peripheral joints in rheumatic diseases. Best Pract Res Clin Rheumatol 2004; 18: 861-879.

7. Freeston JE, Bird P, Conaghan G. The role of MRI in rheumatoid arthritis: research and clinical issues. Curr Opin Rheumatol 2009; 21: 95-101.

8. Narvaez JA, Narvaez J, De Lama E, De Alben M. MR imaging of early rheumatoid arthritis. RadioGraphics 2010; 30; 143-163.

9. Axelsen R, Axelsen MB, Ejbjerg BJ, et al. Differentiation between early rheumaroid arthritis patients nd healthy persons by conventional and dynamic contrast-enhanced magnetic resonance imaging. Scand J Rheumatol 2014; 43: 109-118.

10. Stomp W, Krabben A, van der Hoijde D, et al. Aiming for a simpler early arthritis MRI protocol: can Gd contrast administration be eliminated? Eur Radiol 2015; 25: 1520-1527.

11. Stomp W, Krabben A, van der Hoijde D, et al. Aiming for a shorter rheumatoid arthritis MRI protocol: can contrast-enhanced MRI replace T2 for the detection of bone marrow edema? Eur Radiol 2014; 24: 2614-2622.

12. Morelli JN, Runge VM, Attenberger U, et al. An Image-based Approach to Understanding the Physics of MR Artifacts. RadioGraphics 2011; 31: 849-866.

13. Hodgson RJ, O'Connor P, Moots R. MRI of rheumatoid arthritis-image quantification for the assessment of disease activity, progression and response to therapy. Rheumatology 2008; 47: 13-21.

14. Singh DR, Chin MSM, Peh WCG. Artifacts in musculoskeletal MR imaging. Semin Musculoskelet Radiol 2014; 18: 12-22.

15. Zhuo J, Gullapalli RP. MR artifacts, safety, and quality control. RadioGraphics 2006; 26: 275-297.

16. Dill T. Contraindications to magnetic resonance imaging. Heart 2008; 94; 943-948;

17. Link TM. Osteoporosis imaging: state of the art and advanced imaging. Radiology 2012; 263: 3-17.

18. Stoller DW (ed.). Magnetic Resonance Imaging in Orthopaedics and Sports Medicine. 3rd Edition. Lippincott Williams and Wilkins, Philadelphia 2007; 1997-2042. 